\title{
Pulse Shape Discrimination and Dark Matter Search with $\mathrm{NaI}(\mathrm{Tl})$ Scintillator at the Laboratoire Souterrain de Modane
}

\author{
G. Gerbier ${ }^{1}$, J. Mallet ${ }^{1}$, L. Mosca ${ }^{1}$, and C. Tao ${ }^{2}$ \\ 1 DSM/DAPNIA/SPP, C.E.A. Saclay, F-91191 Gif-sur-Yvette, France \\ 2 CPPM,IN2P3/CNRS and Université Aix-Marseille II, 163 av. de Luminy, case 907, \\ F-13288 Marseille Cedex 09, France
}

\begin{abstract}
Underground data for WIMP search have been obtained from a 83 days rum of a $10 \mathrm{~kg} \mathrm{NaI}(\mathrm{Tl})$ detector in a low activity environment at the Laboratoire Souterrain de Modane (LSM). Emphasis is put in this paper on the understanding of the $2-5 \mathrm{keV}$ energy region, crucial both at establishing limits and at analyzing annual modulation of Spin Independent WIMPs. Part of the PMT noise, dominant in that energy region could induce errors in the results of the modulation analysis. Pulse shape analysis of the data at higher energies have shown an unexpected population of "U" events, also observed by another experiment at the same rate. Additional data obtained at LSM on "U" events are reported. A possible interpretation of these events is related to alpha decays of Radon descendants near the surface of the crystal.
\end{abstract}

\section{Introduction}

The results of the Saclay/Lyon groups $\mathrm{NaI}(\mathrm{Tl})$ experiment performed at the Laboratoire Souterrain de Modane have been recently published in [1]. The goal of the contribution to this conference is first to underline some basic properties of $\mathrm{NaI}(\mathrm{Tl})$ which determine the way its sensitivity depends on the type of the WIMP interaction, Spin Independent (SI) or Spin Dependent (SD), second to show the importance of the low energy spectrum shape in the SI case, in particular the role of the PMT noise in the $2-5 \mathrm{keV}$ region, third to discuss the origin of an unexpected "Bump/U event" population shown by the pulse shape analysis.

\section{Sensitivity of $\mathrm{NaI}(\mathrm{Tl})$ to SI and SD Interacting WIMPs}

The sensitivity of NaI(Tl) to SI and SD WIMP couplings depends on various experimental and theoretical parameters which determine the shape of the expected energy spectra of the WIMP interactions with $\mathrm{Na}$ and I recoils.

First, the scintillation light efficiency or "quenching factor" is smaller for Iodine recoils ( $8 \%$ ) than for Sodium recoils $(25 \%)$. It induces a shrinking of the recoil spectra from Iodine at low energies.

Second, the high mass of the Iodine nucleus induces a loss of coherence in the interaction with a WIMP at the typical energy transfers involved, leading to a reduction of the cross section at the highest energies. 
A third important parameter is the relative height of the two I and $\mathrm{Na}$ recoil energy spectra in SD and SI cases. This parameter depends on the relative cross sections on $\mathrm{Na}$ and I nuclei. For the SI case, the expected cross section is roughly proportional to the square of the atomic mass so the cross section on Iodine largely dominates, while for the SD case, it depends on the nuclear spin factors.

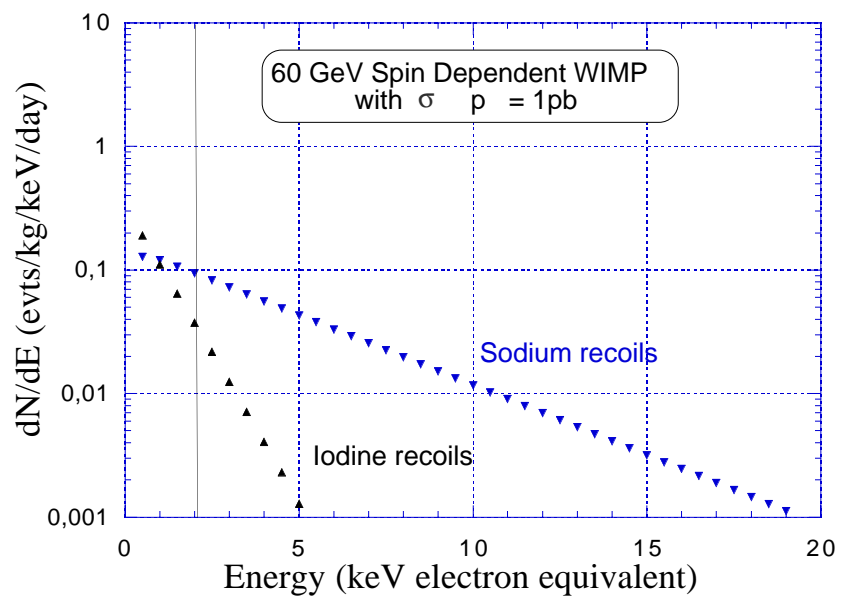

Fig. 1. Energy spectra of I and Na recoils of a $60 \mathrm{GeV}$ SD WIMP signal in NaI(Tl), taking into account all experimental effects

The calculations of the expected energy spectra shown in figures 1 and 2 for a typical $60 \mathrm{GeV}$ mass WIMP include all the above theoretical and experimental parameters. It is clear that above the experimental energy threshold of $2 \mathrm{keV}$, a $\mathrm{NaI}(\mathrm{Tl})$ experiment is sensitive to $\mathrm{Na}$ recoils in the $\mathrm{SD}$ case (figure 1 ). For the SI case the Iodine recoils dominate over the Na recoils below $6 \mathrm{keV}$ by more than one order of magnitude (figure 2) and experiments with a typical "flat" spectrum at say 1 to 2 evts $/ \mathrm{kg} / \mathrm{keV} /$ day will be sensitive to Iodine recoils. The best sensitivity is then obtained at the lowest energy bin (figure 3 ).

Another experimental proccedure involved in the determination of the sensitivity is the Pulse Shape Discrimination (PSD) which allows to reduce the background of electrons through the analysis of the decay time of the scintillation pulses. The reduction factors have been measured by different groups, depend on the temperature, on the light collection efficiency, but all agree that this analysis is only statistical (not event by event) and possible only above 4 $\mathrm{keV}$ electron equivalent energy. Typical statistical rejection factors of the background for an experiment at room temperature of $1000 \mathrm{~kg}$.day are shown on figure 3 . They increase as the square root of the exposure.

Also reported on figure 3 are the typical experimental spectra obtained by the present experiment and the DAMA experiment [2]. 


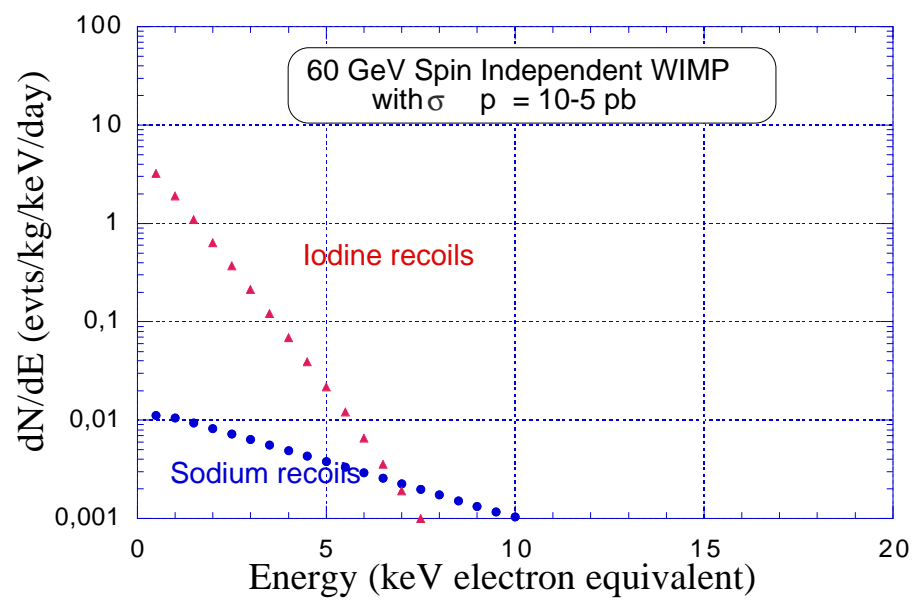

Fig. 2. Energy spectra of I and Na recoils of a $60 \mathrm{GeV}$ SI WIMP signal in NaI(Tl), taking into account all experimental effects

It can be noticed that the decrease with energy of the Iodine recoil spectrum is faster than the increase of the reduction factors. This means that the point of maximum sensitivity using the PSD is the lowest energy point, that is at $5 \mathrm{keV}$. From figure 3, it can be seen that this data point has about the same sensitivity as the $2.5 \mathrm{keV}$ point without PSD. In the DAMA experiment [2], which has a luminosity 16 times higher so a rejection factor 4 times higher, the best sensitivity is still at $2.5 \mathrm{keV}$-without PSD- because of the falling spectrum at the lowest energy.

In both cases the PSD does not help improving the sensitivity, which is then determined by the experimental rate at the lowest energy, i.e. at $2.5 \mathrm{keV}$. This emphasizes the importance of understanding the behaviour of the low energy spectrum, discussed in the next section.

\section{Set-Up}

The setup used at LSM consisted of a 10 by 10 by $25 \mathrm{~cm}$ long NaI(Tl) low activity crystal viewed by two EMI 3 inch low activity PMT through $10 \mathrm{~cm}$ quartz light guides. The detector was installed inside a low activity ancient lead shield enclosed in a plexiglass box continuously flushed with nitrogen to avoid radon contamination. The presently described detector module is similar to the ones used in the DAMA experiment in size, crystal growing method, manufacturer, housing, light guides, PMT type. The measured photoelectron yield was about $7 \mathrm{pe} / \mathrm{keV}$. All pulses above $1.8 \mathrm{keV}$ were digitised at $200 \mathrm{Mhz}$ and recorded on tape. The live time measurement was 83 days. 


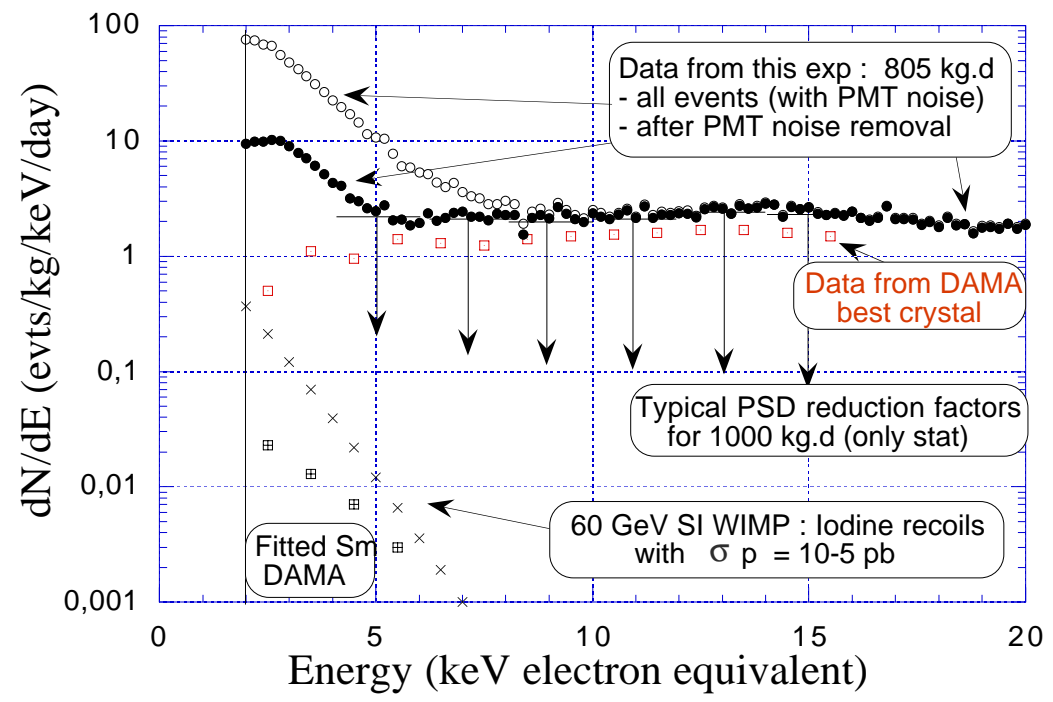

Fig. 3. Energy spectra of a) expected signal in NaI(Tl) from a $60 \mathrm{GeV} \mathrm{SI} \mathrm{WIMP,} \mathrm{b)} \mathrm{of}$ the experimental spectra measured by this experiment (raw and cleaned up) and the DAMA one of their best crystal, together with typical reduction factors obtained with PSD c) of the Sm annual modulation signal of DAMA. See text for details

\section{Low Energy Spectrum}

On figure 3 is shown the obtained raw spectrum of a subsample of the total exposure.

\subsection{PMT Noise}

The 2 to $5 \mathrm{keV}$ energy region is dominated by PMT noise pulses. The total rate in the $2-3 \mathrm{keV}$ bin is 80 evts $/ \mathrm{kg} / \mathrm{keV} /$ day. They have characteristic sharper shapes than scintillation light and strong asymetry between the two PMT signals. Figure 4 shows a scatter diagram of a "sharpness" parameter discriminating between the PMT noise and true NaI(Tl) pulses as a function of energy for all the recorded pulses.

By applying cuts on this parameter and on asymetry, PMT noise can be mostly rejected. We found that we keep $0.5 \%$ of PMT noise in the $2-3 \mathrm{keV}$ bin, that is 0.4 evts $/ \mathrm{kg} / \mathrm{keV} /$ day. On the other hand the efficiency for keeping the "good" scintillation pulses is $75 \%$ in this same bin of energy and rises up to 99 $\%$ at $4 \mathrm{keV}$.

\subsection{Comment on the Annual Modulation Search}

This figure of the contamination of PMT pulses is very important since most of the annual modulation signal claimed by the DAMA group [5] comes from 
the very first $\mathrm{keV}$ energy bin. Varying with the cuts applied in the analysis, a contamination by PMT noise is unavoiddably present among the scintillation pulses. It thus should be prooved that this noise is stable and does not modulate. This was not specifically considered in ref [5].

On the other hand, it can be observed on figure 3 that the energy spectrum shape of the claimed Sm signal is indeed very close to the initial PMT noise shape spectrum.

\subsection{Comment on the Limit Extracted in the WIMP Sigma Mass Plane}

Coming back to the spectrum obtained after cleaning up of the PMT noise, an accumulation is observed at $3 \mathrm{keV}$ (figure 4) in the $\mathrm{NaI}(\mathrm{Tl})$ pulse region, and explains the rising up of spectrum (shown on figure 3) at the lowest energy, up to $10 \mathrm{evts} / \mathrm{kg} / \mathrm{kev} /$ day at $2.5 \mathrm{keV}$. This contrasts with the spectrum observed by DAMA in particular for their best crystal [2] (see figure 3), which is roughly at the same level above $10 \mathrm{keV}$, but lowers to $0.5 \mathrm{evts} / \mathrm{kg} / \mathrm{keV} /$ day at $2.5 \mathrm{keV}$.

This mostly explains the difference in sensitivity of the two experiments in the cross section mass exclusion diagram for SI WIMP : of about $1310^{-5} \mathrm{pb}$ for this experiment as compared to $0.510^{-5} \mathrm{pb}$ for DAMA at a WIMP mass of 100 $\mathrm{GeV}$.

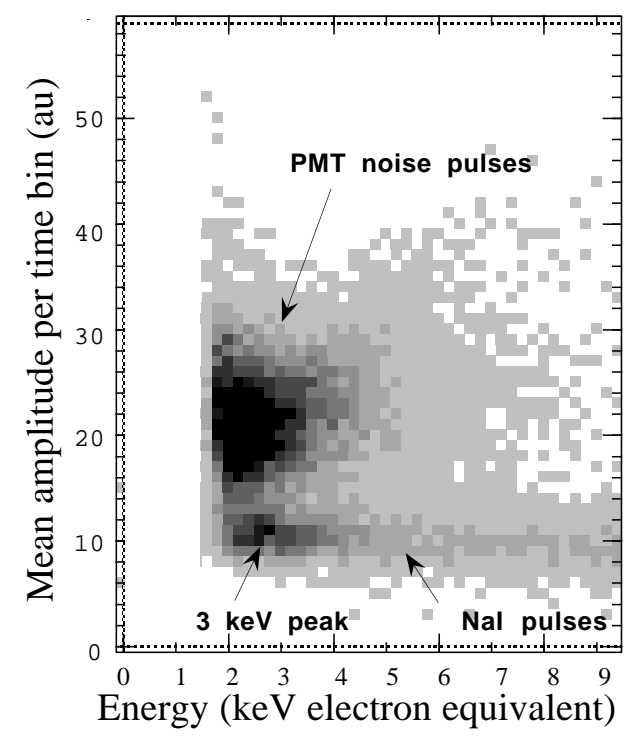

Fig. 4. Scatter plot of the mean amplitude per time bin as a function of energy for data events before appyling any software cuts. The $3 \mathrm{keV}$ peak can clearly be seen in the $\mathrm{NaI}(\mathrm{Tl})$ pulses region 
However, the falling down spectrum below $3 \mathrm{keV}$ of DAMA is hard to reconcile with the expected resolution of the detector [3] and the usual behaviour of electromagnetic background spectra, typically flat or rising up with decreasing energy.

\section{Pulse Shape Analysis and U Event Population}

Pulse shape analysis of the $805 \mathrm{~kg}$.day data, at energies higher than $10 \mathrm{keV}$, have shown an unexpected population of "U" events with even faster decay times than nuclear recoil pulses [1]. UKDMC has observed the same kind of events, the "Bump" events [4] and similar total rates of about 3 evts $/ \mathrm{kg} /$ day and differential energy spectra were observed, between the present experiment and the $6 \mathrm{~kg}$ data, see figure 5 .

Two other $10 \mathrm{~kg}$ crystals, manufactured by BICRON twelve years ago, have been recently measured at LSM. Measured U event spectra are shown also on figure 5. They are again very similar to the ones previously obtained.

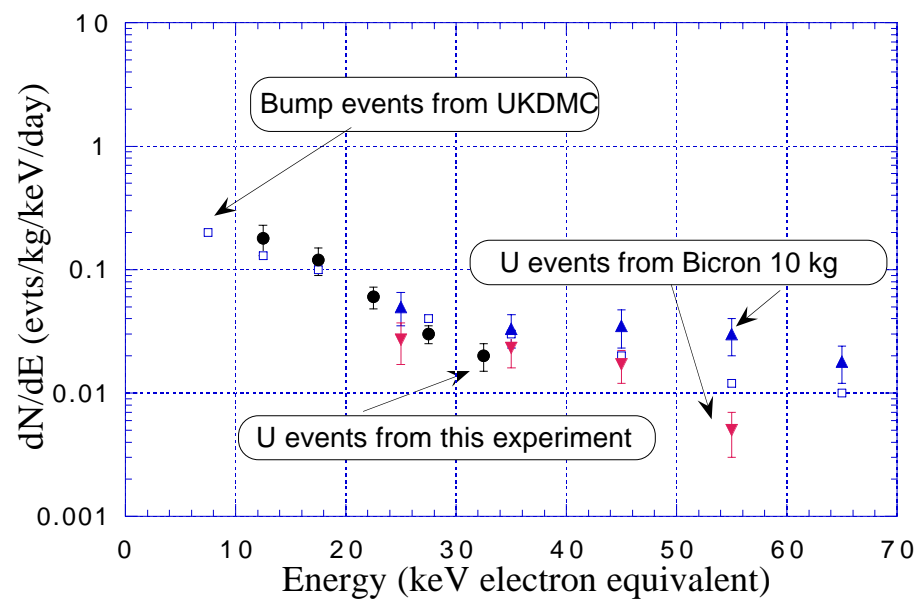

Fig. 5. Energy spectra of U events (from this experiment), Bump events from UKDMC result [4], and more recent results from Bicron crystals measured at LSM

The most likely explanation is related to the existence of surface events. We found that $\mathrm{X}$-rays exhibited faster decay times than Compton. This was not observed by the UKDMC group with enuncapsulated crystals. A possible explanation is that the Modane calibration was done at a single spot, through a thin window, where the $\mathrm{NaI}(\mathrm{Tl})$ surface may have been deteriorated and the response could be different from the rest of the surface.

On the other hand, the UKDMC group has found that alpha particles, generated by a source outside the crystal and slowed down through absorbers, indeed 
give faster decay times than recoils at relevant energies, but not as fast as $\mathrm{U}$ events [6].

Indeed, a more likely explanation consists in a radon surface contamination. Recoiling nuclei from descendants of radon implant slightly inside the crystal. Subsequent alpha decays give rise to recoiling nucleus and escaping $\mathrm{MeV}$ alpha. Then the low energy $U$ events are the result of the sum of the deposited energy by the recoiling nucleus ( $100 \mathrm{keV}$ recoil gives a $8 \mathrm{keV}$ equivalent electron energy) and a part of the deposited energy by the alpha before it escapes the crystal (up to $70 \mathrm{keV}$ ).

However, preliminary calculations by the UK group [7] show that the needed amount of radon accumulated at the surface between the cutting and the sealing of the crystal seems much higher than what can be reasonably inferred from the known way of manufacturing low activity detectors.

Even if this explanation holds, it does not account for the surprising similarity of spectra between crystals of very different origins and manufacturers.

An experimental investigation is led together with the UKDMC group to disentangle this issue. Various crystals have been tested. In particular, an experiment led by the UKDMC group is currently under way to expose a bare crystal to a radon contamination, and measure the shape of the pulses and the energy spectrum of such events.

\section{References}

1. G. Gerbier et al.: Astropart. Phys. 11, 287 (1999)

2. R. Bernabei et al.: Phys. Lett. B 450, 448 (1999)

3. R. Gaitskell: these proceedings

4. P.F. Smith et al.: Phys. Rep. 307, 275 (1998)

5. R. Bernabei et al.: Phys. Lett. B 480, 23 (2000)

6. V. Kudryavtsev et al.: Phys. Lett. B 452, 167 (1999)

7. N.J.C. Spooner: these proceedings 